\title{
Peroxisome proliferator-activated receptor $\gamma$ agonist effect on rheumatoid arthritis: a randomized controlled trial
}

Michelle J Ormseth ${ }^{1 *}$, Annette M Oeser ${ }^{1,2}$, Andrew Cunningham ${ }^{2}$, Aihua Bian ${ }^{3}$, Ayumi Shintani $^{3}$, Joseph Solus ${ }^{1}$, S Bobo Tanner ${ }^{1}$ and C Michael Stein ${ }^{1,2}$

\begin{abstract}
Introduction: Rheumatoid arthritis (RA), a chronic inflammatory disease, is associated with insulin resistance. Experimental evidence indicates that the relationship between insulin resistance and inflammation is bidirectional: Inflammation promotes insulin resistance, and insulin resistance promotes inflammation. Therefore, we examined the hypothesis that pioglitazone, a thiazolidinedione peroxisome proliferator-activated receptor $\gamma$ agonist, would decrease inflammation and disease activity and improve insulin resistance in patients with RA.

Methods: In a single-center, randomized, double-blind, placebo-controlled crossover study patients with RA $(N=34)$ receiving stable therapy were randomized to also receive either pioglitazone $45 \mathrm{mg}$ daily $(n=17)$ or matching placebo $(n=17)$ for eight weeks. This was followed by a four-week washout period and alternative treatment for eight weeks. Outcomes included change in Disease Activity Score in 28 joints (DAS28) score, individual components of the DAS28 score and homeostatic model assessment for insulin resistance (HOMA). Intention-to-treat analysis and linear mixed-effects models were used.
\end{abstract}

Results: Patients had a mean ( \pm SD) age of 51 ( \pm 14.2$)$ years, 82.4\% were female and baseline DAS28 high-sensitivity C-reactive protein (DAS28-CRP) was $4.58( \pm 1.1)$ units. Addition of pioglitazone was associated with a 9.3\% reduction (95\% confidence interval $(C l)=0.17 \%$ to $17.6 \%)$ in DAS28-CRP $(P=0.046)$, but no significant change in DAS28 erythrocyte sedimentation rate (DAS28-ESR) $(P=0.92)$. There was a $10.7 \mathrm{~mm}(95 \% \mathrm{Cl}=0.4$ to $20.9 \mathrm{~mm})$ improvement in patient-reported global health $(P=0.042)$, a $48.6 \%$ decrease $(95 \% \mathrm{Cl}=27.6 \%$ to $63.5 \%)$ in CRP $(P<0.001)$ and a $26.4 \%$ decrease $(95 \% \mathrm{Cl}=3.7 \%$ to $43.8 \%)$ in insulin resistance as measured by HOMA $(P=0.025)$, but no significant reduction in swollen or tender joint count or in ESR (all $P>0.05$ ). Lower-extremity edema was more common during pioglitazone treatment (16\%) than placebo (0\%).

Conclusion: Addition of pioglitazone to RA therapy improves insulin resistance and modestly reduces RA disease activity measured by DAS28-CRP and two of its components, including patient-reported global health and CRP, but not DAS28-ESR or ESR.

Trial registration: NCT00763139

\footnotetext{
* Correspondence: michelle.ormseth@vanderbilt.edu

'Division of Rheumatology, Department of Medicine, Vanderbilt University, Nashville, TN, USA

Full list of author information is available at the end of the article
} 


\section{Introduction}

Patients with rheumatoid arthritis (RA), a chronic systemic inflammatory disease, have an increased incidence of premature atherosclerosis, even after adjusting for traditional cardiovascular risk factors [1]. We have reported previously that patients with RA have more than a twofold increased incidence of insulin resistance and that this was associated with inflammation, increased disease activity and coronary atherosclerosis [2,3]. Moreover, insulin resistance has been associated with increased inflammation in populations without RA [4-8]. Additional evidence that inflammation can promote insulin resistance is provided by studies in animals and humans [9-11]. For example, in diabetic rats, administration of tumor necrosis factor $\alpha$ (TNF- $\alpha$ ) caused hyperglycemia [12], and, in obese rats, neutralization of TNF- $\alpha$ improved insulin resistance [13]. In humans, treatment of active RA, even with corticosteroids, a class of drug which would be expected to increase insulin resistance, can decrease insulin resistance, presumably by decreasing inflammation [14].

Recent evidence indicates that, just as inflammation may result in insulin resistance, hyperinsulinemia may increase inflammation. For example, in vitro studies have shown that glucose and insulin-like growth factors augment the secretion of inflammatory cytokines in mononuclear cells $[15,16]$. Also, in humans, euglycemic hyperinsulinemia amplifies and prolongs the interleukin 6 (IL-6) response to endotoxin [17]. Thus, there appears to be a bidirectional relationship between inflammation and hyperinsulinemia.

Concordant with the finding that insulin resistance may promote inflammation, therapies which improve insulin resistance may decrease inflammation. Thiazolidinediones, selective ligands of the nuclear transcription factor peroxisome proliferator-activated receptor $\gamma$ (PPAR- $\gamma$ ), are insulin sensitizers, even in individuals without diabetes [18]. Studies in animals, including a model of arthritis, as well as in patients with psoriatic arthritis and lupus, have shown that, in addition to improving insulin sensitivity, thiazolidinediones decrease inflammation [19-28].

We therefore performed this study to test the hypothesis that pioglitazone would decrease disease activity, inflammation and insulin resistance in patients with RA.

\section{Methods}

\section{Patients}

Patients older than 18 years of age who met the 1987 American Rheumatism Association criteria for RA [29] with moderate disease activity (at least three tender and three swollen joints) and no change in immunomodulating or anti-inflammatory therapy in the past one month were eligible for enrollment. Patients were excluded if they had active cancer other than skin cancer, HIV, heart failure, severe edema, diabetes mellitus requiring drug therapy, aspartate aminotransferase or alanine aminotransferase greater than twice the upper limit of normal, major surgery within the previous three months, untreated osteoporosis, allergy to pioglitazone, previous organ or bone marrow transplant or severe comorbid conditions likely to compromise survival or study participation. Patients were also excluded if they were receiving dialysis, taking gemfibrozil or rifampin or unwilling or unable to cooperate. Additionally, patients were excluded they were pregnant, or if they had childbearing potential and would not agree to affective birth control. After reviewing reports about the possible association between pioglitazone and bladder cancer [30,31], we excluded patients with a history of bladder cancer or precancerous bladder lesions. The study was approved by the Vanderbilt University Institutional Review Board, and all participants gave their written informed consent.

\section{Study design}

This trial was a single-center, randomized, double-blind, placebo-controlled crossover study with washout. We chose to use a crossover study design because this model increases the power to detect a difference with small sample sizes by reducing between-patient variability and the effect of confounders when each patient serves as his or her own control. Patients were randomly assigned to receive pioglitazone $45 \mathrm{mg}$ or matching placebo daily for eight weeks in combination with unchanged doses of their baseline disease-modifying antirheumatic drug (DMARD) therapy. This treatment was followed by a fourweek washout period, then a second eight-week treatment period with the alternative therapy (Figure 1). Study visits occurred at screening, at baseline (week 0 ) and every four weeks through week 20 or withdrawal from the study. Patients were randomly assigned to a treatment sequence (placebo or pioglitazone first) according to a randomization table generated using a permuted block randomization scheme prepared by the study statistician. Pioglitazone or

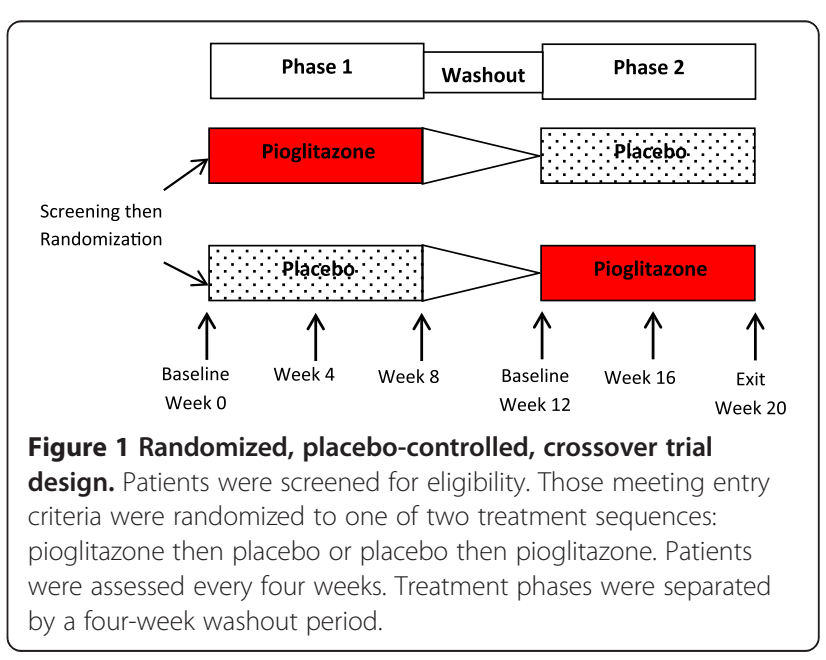


identical placebo capsules were dispensed by the Vanderbilt University investigational pharmacy. If a change in RA disease status warranted any change in DMARD or corticosteroid therapy, the patient was withdrawn from the study.

At the baseline (week 0) visit, the following clinical details were obtained from the patient and the medical records: height, weight, waist and hip measurements; blood pressure; current and past smoking history; personal and family history of cardiovascular disease; medication use, including current and past DMARD use; current and cumulative prednisone therapies; and cardiovascular therapies.

At baseline and at each subsequent visit, participants were asked to temporarily omit medications, including nonsteroidal anti-inflammatory drugs (NSAIDs) and corticosteroids, on the morning of the study day. Patients were studied after at least 6 hours of fasting and after 30 minutes of supine rest at approximately the same time of day. A physical examination was performed, and RA disease activity indices, including 28 tender and swollen joint counts, were measured and venous blood was drawn for laboratory assessments. Urine and/or serum pregnancy tests were performed at each study visit in females with reproductive potential.

Laboratory examinations comprising a complete blood count; creatinine, glucose and liver function tests (LFTs); high-sensitivity $\mathrm{C}$-reactive protein (CRP) and erythrocyte sedimentation rate (ESR) were performed by the hospital clinical laboratory staff. Fasting plasma insulin levels were measured at the Vanderbilt Hormone Assay Core Laboratory by radioimmunoassay. Serum cytokines, including tumor necrosis factor $\alpha$ (TNF- $\alpha$ ) and IL-6, were measured by multiplex enzyme-linked immunosorbent assay (EMD Millipore, Billerica, MA, USA) in duplicate in batches with samples from each time point in a given patient run together on the same plate with appropriate controls and blocking agents. All measurements were taken at the beginning and end of each treatment arm.

\section{Outcome measures}

The primary outcome was change in Disease Activity Score in 28 joints (DAS28) score (calculated as DAS28$\mathrm{CRP}=0.56 * \operatorname{sqrt}\left(\right.$ tender $\left._{28}\right)+0.28 * \operatorname{sqrt}\left(\right.$ swollen $\left._{28}\right)+0.70$ * $\ln (\mathrm{CRP})+0.014$ " global health $(\mathrm{GH})$ and DAS28-ESR = $0.56 * \operatorname{sqrt}\left(\right.$ tender $\left._{28}\right)+0.28 * \operatorname{sqrt}\left(\right.$ swollen $\left._{28}\right)+0.70 * \ln$ $(\mathrm{ESR})+0.014 * \mathrm{GH})$. Other prespecified outcomes included individual components of the DAS28 score, including tender and swollen joint count, patient-reported GH based on 1- to 100-mm Visual Analogue Scale (VAS) rating and acute-phase reactants (CRP, ESR); level of inflammatory cytokines IL- 6 and TNF- $\alpha$; change in functional capacity determined using the modified Health Assessment Questionnaire (MHAQ) [32]; and change in insulin resistance determined by the homeostatic model assessment for insulin resistance (HOMA), calculated as (fasting glucose [in $\mathrm{mmol} / \mathrm{L}] \times$ fasting insulin $[$ in $\mu \mathrm{U} / \mathrm{ml}]$ )/ 22.5 [33].

\section{Statistical analysis}

Sample size was estimated using a paired $t$-test and assuming a baseline DAS28 score of $5.0 \pm 1.0$ units [34,35]. A sample size of 32 patients would provide $90 \%$ power to detect a 0.6-unit greater decrease in DAS28 score in patients taking pioglitazone compared to placebo after excluding dropouts (defined as having no follow-up study visits). We initially aimed to enroll 36 patients with the assumptions that 4 patients would drop out (defined as not returning for any visits) and 32 patients would provide data for analysis. We stopped enrollment after patient 34, however, because there had been no dropouts.

Descriptive statistics are presented as frequencies and percentages (\%) for categorical variables and means with standard deviations (mean $\pm \mathrm{SD}$ ) for continuous variables. Linear mixed-effects models were used to quantify the effect of pioglitazone on outcomes. Data gathered from all study visits from all patients, including those who exited the study early and those who were withdrawn, were used in the analysis. The linear mixedeffects models were fitted for all outcomes separately. These modeled the fixed effects of the baseline value of the outcome, treatment regimen, week and product of week and treatment regimen. Random intercepts were used to model the variability between patients. For all the primary and secondary outcomes, the carryover effect was assessed by comparing the means of the baseline outcome measurements between patients who received pioglitazone in the first phase followed by placebo and those who received placebo in the first phase followed by pioglitazone using linear mixed-effects models with random intercepts for between-patient variations. Because we used a two-arm crossover design in this study, data were pooled between the two treatment phases after finding no carryover effect, and we report between-group differences at the end of study time points (week 8 and week 20) using general contrast of regression coefficients in the mixed-effects models.

To assess whether pioglitazone's effect on HOMA mediated the effect on disease indices such as DAS28-CRP, we used mixed-effect models to examine attenuation in the effect of the treatment by adjustment for the change in HOMA [36]. The values for DAS28-CRP, DAS28-ESR, CRP, ESR, MHAQ, IL-6, TNF- $\alpha$ and HOMA were all natural logarithm-transformed to improve normality in the residuals. Thus, changes in these outcomes were backtransformed and presented as percentage changes. Statistical analyses were performed using the $\mathrm{R}$ Project for 
Statistical Computing version 2.15.1 software (http://www. r-project.org/). A two-sided significance level of 5\% was required to consider the data as statistically significant.

\section{Results \\ Patients}

The study was performed from May 2009 to April 2012. Thirty-four patients with RA were studied (Figure 2). Patients had a mean age $( \pm \mathrm{SD})$ of 51.0 years $( \pm 14.2)$, $82.4 \%$ were female and the baseline mean DAS28-CRP was 4.58 units $( \pm 1.1)$. Of the 28 patients with available rheumatoid factor (RF) or anti-citrullinated peptide antibody (ACPA) test results, 24 (85.7\%) were RF- or ACPApositive. Of the 21 patients with X-rays, 15 (71.4\%) had erosive disease. The baseline characteristics of the patients are shown in Table 1.

\section{Effect of pioglitazone on rheumatoid arthritis disease indices and inflammatory markers}

The addition of pioglitazone was associated with a 9.3\% decrease $(95 \%$ confidence interval $(95 \% \mathrm{CI})=0.17 \%$ to $17.6 \%)$ in DAS28-CRP $(P=0.046)$ compared to placebo; however, the effect of pioglitazone on DAS28-ESR was not statistically significant $(P=0.92)$ (Table 2 and Figure 3). Individual components of the DAS28 score were evaluated. Pioglitazone was associated with a $10.7-\mathrm{mm}$ improvement ( $95 \% \mathrm{CI}=0.4$ - to $20.9-\mathrm{mm}$ ) in patient-reported GH on the VAS $(P=0.042)$ and a $48.6 \%$ decrease in CRP $(95 \% \mathrm{CI}=27.6 \%$ to $63.5 \%)(P<0.001)$, but no significant change in swollen joint count $(P=0.77)$, tender joint count $(P=0.54)$ or ESR $(P=0.32)$ compared to placebo (Table 2). There was no significant change in MHAQ score $(P=0.51)$ with pioglitazone use (Table 2$)$. Use of pioglitazone was associated with a $67.0 \%$ reduction in IL-6 $(95 \% \mathrm{CI}=28.2 \%$ to $84.6 \%)(P=0.01)$, but no significant change in TNF- $\alpha(P=0.71)$ (Table 2).

\section{Effect of pioglitazone on insulin resistance and relationship to disease indices}

Use of pioglitazone was associated with a $26.4 \%$ decrease ( $95 \% \mathrm{CI}=3.7 \%$ to $43.8 \%$ ) in insulin resistance as measured by HOMA $(P=0.025)$ (Table 2$)$. To determine whether improvement in insulin resistance caused the improvements in disease activity indices, we compared the results

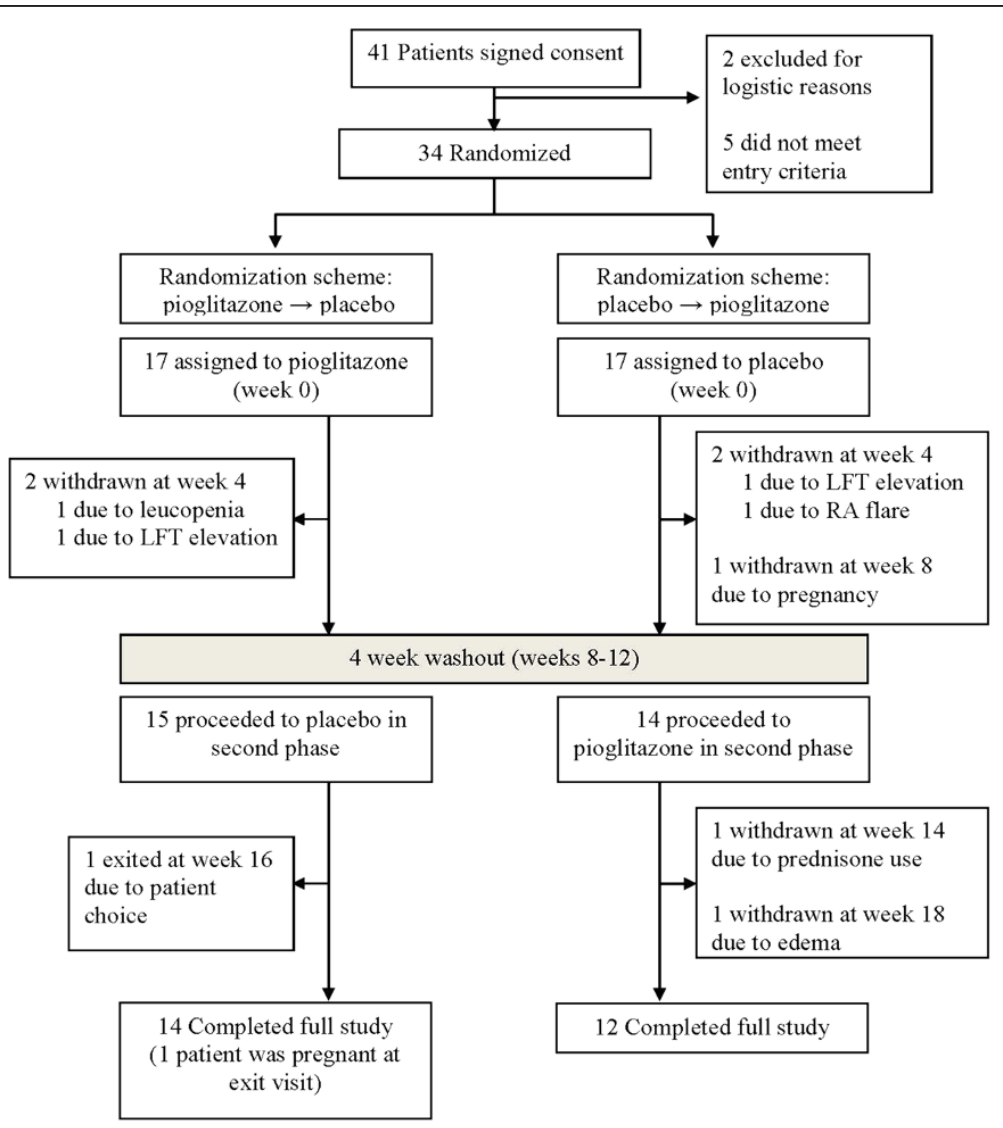

Figure 2 Randomization and follow-up of study participants. A total of 34 patients with rheumatoid arthritis (RA) were enrolled in the study. There was at least one follow-up visit for all patients. A total of 31 patients were exposed to pioglitazone, and 32 were exposed to placebo. LFT, liver function test. 
Table 1 Baseline demographics and clinical characteristics of patients $^{\mathrm{a}}$

\begin{tabular}{lc}
\hline Demographics and characteristics & Data \\
\hline Demographics and anthropomorphic measures & \\
Age (years) & $51.0( \pm 14.2)$ \\
Females (\%) & $82.4 \%(28)$ \\
Race, Caucasians (\%) & $85.3 \%(29)$ \\
Weight (kg) & $77.3( \pm 18.1)$ \\
BMl (kg/m²) & $28.28( \pm 6.04)$ \\
Disease-related and laboratory indices & \\
DAS28-CRP (units) & $4.58( \pm 1.10)$ \\
Tender joints ( $n)$ & $9.68( \pm 6.96)$ \\
Swollen joints ( $n)$ & $8.38( \pm 4.66)$ \\
VAS (mm) & $43.0( \pm 28.5)$ \\
CRP (mg/dl) & $12.69( \pm 24.57)$ \\
ESR (mm/h) & $22.8( \pm 21.3)$ \\
HOMA (units) & $3.11( \pm 2.68)$ \\
Current medication use & \\
Methotrexate (\%) & $70.6 \%(24)$ \\
Leflunomide (\%) & $14.7 \%(5)$ \\
Sulfasalazine (\%) & $2.9 \%(1)$ \\
Hydroxycholorquine (\%) & $17.6 \%(6)$ \\
Biologic (\%) & $52.9 \%(18)$ \\
Corticosteroid (\%) & $52.9 \%(18)$ \\
\hline
\end{tabular}

${ }^{a}$ Data are presented as mean $( \pm S D)$ for continuous data and percentage (number) for categorical variables. BMI, body mass index; CRP, high-sensitivity C-reactive protein; DAS28-CRP, Disease Activity Score in 28 joints highsensitivity C-reactive protein; ESR, erythrocyte sedimentation rate; HOMA, homeostatic model assessment of insulin resistance; VAS, Visual Analogue Scale for Global Health.

of our above-described models with the results after additional adjustment for HOMA. There was no attenuation of effect after adjustment for HOMA: DAS28-CRP, $-9.8 \%$ (95\% $\mathrm{CI}=-18.1 \%$ to $-0.6 \% ; P=0.037) ; \mathrm{CRP},-48.9 \%$ (95\% $\mathrm{CI}=-66.2$ to $-22.8 \% ; P=0.001)$; VAS, $10.5 \mathrm{~mm}$ (95\% CI $=-19.6$ to $-1.35 ; P=0.002)$; and IL-6, $-71 \%(95 \%$ $\mathrm{CI}=-87.8$ to $-31.8 \% ; P=0.005)$. Thus, we determined that there was no statistical evidence that pioglitazone's effect on HOMA mediated changes in outcome measures of inflammation.

\section{Drug side effects}

Treatment with pioglitazone was generally well-tolerated. Adverse effects related to the drug included lowerextremity edema in five patients (16\%) compared to none among those taking placebo. Four patients were withdrawn during the pioglitazone arms of the study (one each due to leukopenia, LFT elevation, new prednisone use and lower-extremity edema). Similarly, four patients were withdrawn during the placebo arms of the study (one each due to LFT elevation, RA flare, unintended pregnancy and patient choice) (Figure 2). Use of pioglitazone was associated with a $0.76 \mathrm{~kg}$ increase $(95 \% \mathrm{CI}=0.10$ to $1.42 \mathrm{~kg})$ in body weight $(P=0.023)$.

\section{Washout and assessment for carryover effect}

There was no carryover effect detected for DAS28-CRP $(P=0.92)$ or other outcomes evaluated (all $P>0.05)$.

\section{Discussion}

The major findings of this study are that the addition of pioglitazone to stable DMARD treatment for RA improves insulin resistance, as measured by HOMA, and modestly reduces RA disease activity, as measured by DAS28-CRP, patient-reported GH and CRP levels. The reductions in DAS28-CRP, CRP and IL-6 levels were independent of improvement in insulin resistance.

Several lines of evidence support the idea that PPAR- $\gamma$ agonists have anti-inflammatory effects. PPAR- $\gamma$ expression in peripheral monocytes and monocyte-derived macrophages in RA patients was inversely correlated with disease activity, and some medications commonly used in the treatment of RA (methotrexate and corticosteroids) increase PPAR- $\gamma$ expression [37]. In animal models of RA, thiazolidinedione treatment reduced synovitis, inflammatory bone loss and bone erosion [23-25]. Moreover, a recent single-blind trial suggested improvement in disease activity in diabetic patients with active RA, but an adequate comparator control was not used; thus, definitive conclusions regarding efficacy could not be made [25]. Additionally, in an open-label trial of patients with psoriatic arthritis, pioglitazone reduced both tender and swollen joint counts [26], and pioglitazone decreased CRP and serum amyloid A and improved insulin sensitivity in patients with uncomplicated systemic lupus erythematosus [27].

Our findings raise the possibility that pioglitazone may have different effects on CRP compared to ESR. CRP is produced in the liver in response to IL-6 [38], whereas ESR is a laboratory phenomenon affected by fibrinogen, immunoglobulin levels and red blood cell count and shape, among other factors [39,40]. Several studies have found that pioglitazone decreased CRP concentrations [19-21], but ESR has not been measured routinely in most clinical trials of pioglitazone or other thiazolidinediones. However, in one study that included nondiabetic patients with known cardiovascular disease, pioglitazone resulted in a nonsignificant trend toward increased ESR [41]. The decrease in IL-6 concentrations we observed after pioglitazone therapy is mechanistically concordant with the reduction in CRP that occurred, but factors driving ESR may not have been altered as much $[42,43]$. In contrast to IL-6, TNF- $\alpha$ concentrations were not significantly affected by pioglitazone, raising 
Table 2 Effect of pioglitazone on rheumatoid arthritis disease activity, inflammation and insulin resistance ${ }^{a}$

\begin{tabular}{|c|c|c|c|c|c|c|}
\hline \multirow[t]{3}{*}{ Measurement } & \multicolumn{2}{|c|}{ Pioglitazone phase } & \multicolumn{2}{|c|}{ Placebo phase } & \multicolumn{2}{|c|}{ Pioglitazone treatment effect } \\
\hline & Baseline & Week 8/week 20 & Baseline & Week 8/week 20 & Effect & $P$-value \\
\hline & (SD) & (SD) & (SD) & (SD) & $(95 \% \mathrm{Cl})$ & \\
\hline \multirow[t]{2}{*}{ DAS28-CRP } & 4.40 & 4.03 & 4.57 & 4.48 & $-9.3 \%$ & 0.046 \\
\hline & $(1.00)$ & $(1.15)$ & $(1.28)$ & $(1.20)$ & $(-17.6$ to $-0.17 \%)$ & \\
\hline \multirow[t]{2}{*}{ DAS28-ESR } & 4.56 & 4.37 & 4.85 & 4.57 & $0.6 \%$ & 0.92 \\
\hline & $(1.39)$ & $(1.28)$ & $(1.56)$ & $(1.63)$ & $(-10.3$ to $12.7 \%)$ & \\
\hline \multirow[t]{2}{*}{ Swollen joints ( $n$ ) } & 6.6 & 6.46 & 8.2 & 7 & 0.3 & 0.77 \\
\hline & $(3.7)$ & $(4.79)$ & $(5.6)$ & $(5.11)$ & $(-1.4$ to 1.9$)$ & \\
\hline \multirow[t]{2}{*}{ Tender joints $(n)$} & 9.6 & 8.54 & 11.5 & 10.35 & -0.7 & 0.54 \\
\hline & $(6.89)$ & $(7.28)$ & $(8.1)$ & $(8.23)$ & $(-2.9$ to 1.5$)$ & \\
\hline \multirow[t]{2}{*}{ VAS (mm) } & 44.9 & 42.04 & 48.0 & 49.46 & -10.7 & 0.042 \\
\hline & $(24.7)$ & (28.39) & $(31.2)$ & $(23.62)$ & $(-20.9$ to -0.4$)$ & \\
\hline \multirow[t]{2}{*}{ CRP (mg/dl) } & 8.1 & 5.02 & 7.7 & 8.25 & $-48.6 \%$ & $<0.001$ \\
\hline & $(11.41)$ & (7.64) & (13.6) & $(10.32)$ & $(-63.5$ to $-27.6 \%)$ & \\
\hline \multirow[t]{2}{*}{ ESR (mm/h) } & 18.5 & 17 & 19.5 & 18.88 & $21.3 \%$ & 0.32 \\
\hline & $(18.2)$ & $(17.06)$ & (20) & $(20.80)$ & $(-16.9$ to $77 \%)$ & \\
\hline \multirow[t]{2}{*}{ IL-6 (pg/ml) } & 5.41 & 2.38 & 8.67 & 6.47 & $-67.0 \%$ & 0.01 \\
\hline & $(7.88)$ & $(4.05)$ & (19.31) & $(14.40)$ & $(-84.6$ to $-28.2 \%)$ & \\
\hline \multirow[t]{2}{*}{ TNF-a (pg/ml) } & 9.90 & 9.50 & 13.40 & 9.71 & $6.0 \%$ & 0.71 \\
\hline & $(10.64)$ & $(10.56)$ & (19.41) & $(7.91)$ & (-22.7 to $46.2 \%)$ & \\
\hline \multirow[t]{2}{*}{ MHAQ (units) } & 0.55 & 0.54 & 0.56 & 0.59 & $-4.3 \%$ & 0.51 \\
\hline & $(0.37)$ & $(0.39)$ & $(0.35)$ & $(0.36)$ & $(-16.2$ to $9.3 \%)$ & \\
\hline \multirow[t]{2}{*}{ HOMA (units) } & 2.83 & 2.44 & 2.38 & 3.11 & $-26.4 \%$ & 0.025 \\
\hline & $(2.50)$ & $(2.08)$ & $(1.75)$ & $(3.47)$ & $(-43.8$ to $-3.7 \%)$ & \\
\hline
\end{tabular}

${ }^{\mathrm{a}}$ Data for each intervention phase (pioglitazone and placebo) at baseline and at week 8/week 20 are presented as mean ( \pm SD). Pioglitazone treatment effect was calculated using linear mixed-effects models and is presented as change or percentage change (95\% confidence interval). CRP, high-sensitivity C-reactive protein; DAS28-CRP, Disease Activity Score in 28 joints high-sensitivity C-reactive protein; ESR, erythrocyte sedimentation rate; HOMA, homeostatic model assessment of insulin resistance; IL-6, interleukin 6; MHAQ, modified Health Assessment Questionnaire; TNF-a, tumor necrosis factor a; VAS, Visual Analogue Scale for Global Health.

the possibility that pioglitazone may have greater effects on IL-6 than on TNF- $\alpha$. These modest effects of pioglitazone suggest it is unlikely to be a helpful add-on therapy for reduction of RA disease activity, particularly when one considers the increased risk for fracture, bladder cancer and heart failure in some individuals [44-46].

As part of our analysis, we set out to define whether changes in insulin sensitivity were related to changes in inflammation because this would provide a mechanistic insight. We found that improvements of DAS28-CRP, CRP and IL- 6 after pioglitazone treatment were independent of improvements in HOMA, suggesting that mechanisms other than improved insulin sensitivity are likely to be involved. These findings are compatible with those of a study of healthy participants showing that rosiglitazone reduced CRP within one day, before changes in insulin sensitivity had occurred [47]. Thus, thiazolidinediones may affect inflammation rapidly, before the effects on insulin resistance have occurred, through mechanisms independent of improved insulin sensitivity [47-51].

Our study has some limitations. This study was relatively small, but it was efficiently designed to study the outcomes of interest. The crossover design has the advantage of comparing changes in the same patient and the disadvantage that subtle, undetected carryover effects may have occurred. Several mean baseline disease activity indices (DAS28-CRP, DAS28-ESR, swollen and tender joint counts, VAS, IL- 6 and TNF- $\alpha$ ) were slightly lower at the start of the pioglitazone phase compared to the baseline of the placebo phase. Though not a significant difference, lower disease activity in patients entering the treatment phase could have biased our results toward the null hypothesis. Also, inflammatory cytokine levels were relatively low even at baseline because patients were receiving fairly effective background 

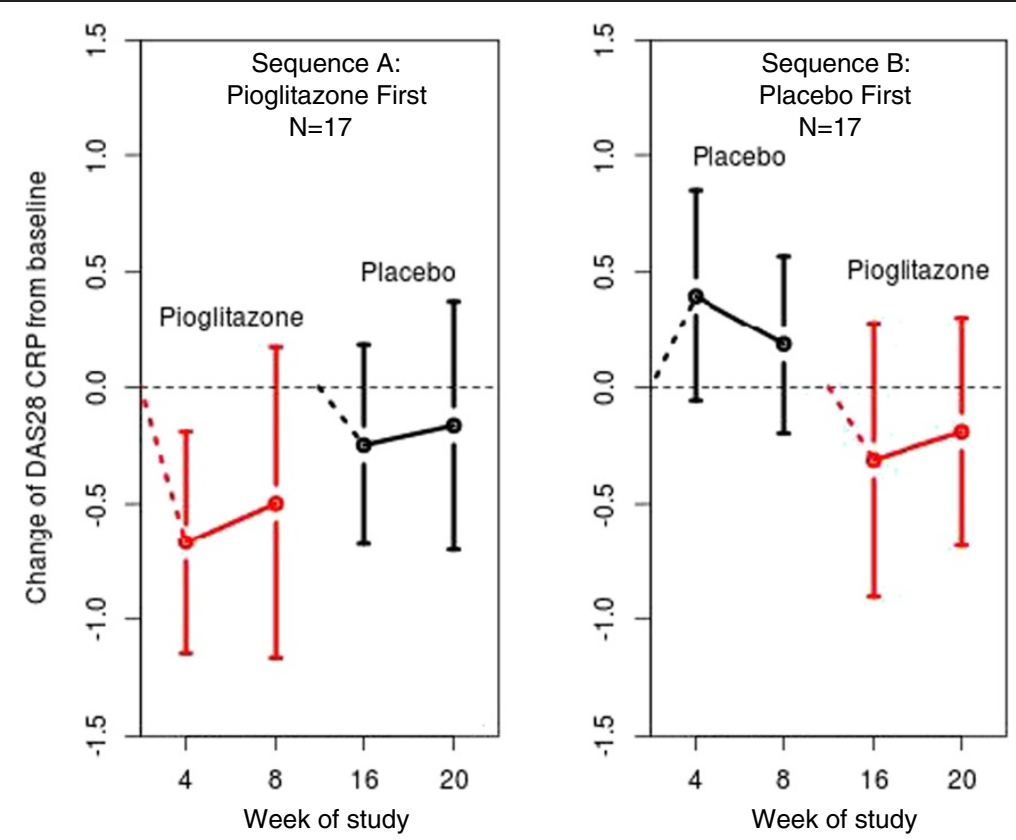

Figure $\mathbf{3}$ Rheumatoid arthritis disease activity measured by Disease Activity Score in $\mathbf{2 8}$ joints score is decreased by pioglitazone. The graph on the left shows the change in Disease Activity Score in 28 joints high-sensitivity C-reactive protein (DAS28 CRP) scores among patients randomized to receive pioglitazone first, then placebo. The graph on the right shows change in DAS28-CRP scores among patients randomized to receive placebo first, then pioglitazone. Red designates patients taking pioglitazone, and black represents those taking placebo. Open circles represent mean change, and bars represent $95 \%$ confidence intervals.

therapy, which also could bias our results toward the null hypothesis. Duration of drug exposure was only eight weeks, which is shorter than most trials of DMARDs and biologics; therefore, we cannot exclude the possibility that extended exposure to pioglitazone may have had different effects on joint counts and overall disease activity.

\section{Conclusion}

Addition of pioglitazone to RA therapy improves insulin resistance and modestly reduces RA disease activity measured by DAS28-CRP and two of its components, patient-reported GH and CRP, but not DAS28-ESR or ESR.

\section{Abbreviations}

ACPA: anti-citrullinated peptide antibody; CRP: high-sensitivity C-reactive protein; DAS: Disease Activity Score; DMARD: disease-modifying antirheumatic drug; ESR: erythrocyte sedimentation rate; GH: global health; HOMA: homeostatic model assessment of insulin resistance; IL-6: interleukin 6; LFT: liver function test; MHAQ: Modified Health Assessment Questionnaire; NSAID: nonsteroidal anti-inflammatory drug; PPAR-ү: peroxisome proliferatoractivated receptor $\gamma$; RA: rheumatoid arthritis; RF: rheumatoid factor;

SD: standard deviation; TNF-a: tumor necrosis factor a.

\section{Competing interests}

The authors declare that they have no competing interests.

\section{Authors' contributions}

MJO performed patient recruitment, study execution, data analysis and interpretation, and drafted the manuscript. AMO and AC performed patient recruitment and study execution. AB performed data analysis. AS participated in study design and performed data analysis. JS performed enzyme-linked immunosorbent assays. SBT performed patient recruitment. CMS conceived the study, established the study design, oversaw study execution, performed data interpretation and oversaw manuscript drafting. All authors read and approved the final manuscript.

\section{Acknowledgements}

This study was funded by the following National Institutes of Health grants: National Institutes of Health National Institute of Arthritis and Musculoskeletal and Skin Diseases (NIAMS) grant P60 AR056116, National Center for Research Resources, now at the National Center for Advancing Translational Sciences, Grants 1 and 2 (UL1 RR024975-01 and UL1 TR000445-06, respectively) and NIAMS grant T32 AR059039.

This trial is registered with ClinicalTrials.gov, NCT00763139 Additionally, the project described was supported by the National Center for Research Resources, Grant UL1 RR024975-01, and is now at the National Center for Advancing Translational Sciences, Grant 2 UL1 TR000445-06.

\section{Author details}

'Division of Rheumatology, Department of Medicine, Vanderbilt University, Nashville, TN, USA. ${ }^{2}$ Division of Clinical Pharmacology, Department of Medicine, Vanderbilt University, Nashville, TN, USA. ${ }^{3}$ Department of Biostatistics, Vanderbilt University, Nashville, TN, USA.

Received: 12 April 2013 Accepted: 20 August 2013

Published: 10 September 2013

\section{References}

1. Chung CP, Oeser A, Raggi P, Gebretsadik T, Shintani AK, Sokka T, Pincus T, Avalos I, Stein CM: Increased coronary-artery atherosclerosis in rheumatoid arthritis: relationship to disease duration and cardiovascular risk factors. Arthritis Rheum 2005, 52:3045-3053.

2. Chung CP, Oeser A, Solus JF, Avalos I, Gebretsadik T, Shintani A, Raggi P, Sokka T, Pincus T, Stein CM: Prevalence of the metabolic syndrome is increased in rheumatoid arthritis and is associated with coronary atherosclerosis. Atherosclerosis 2008, 196:756-763. 
3. Chung CP, Oeser A, Solus JF, Gebretsadik T, Shintani A, Avalos I, Sokka T, Raggi $P$, Pincus T, Stein CM: Inflammation-associated insulin resistance: differential effects in rheumatoid arthritis and systemic lupus erythematosus define potential mechanisms. Arthritis Rheum 2008 58:2105-2112

4. McMillan DE: Increased levels of acute-phase serum proteins in diabetes. Metabolism 1989, 38:1042-1046.

5. Katsuki A, Sumida Y, Murashima S, Murata K, Takarada Y, Ito K, Fujii M, Tsuchihashi K, Goto H, Nakatani K, Yano Y: Serum levels of tumor necrosis factor- $a$ are increased in obese patients with noninsulin-dependent diabetes mellitus. J Clin Endocrinol Metab 1998, 83:859-862.

6. Festa A, D'Agostino R Jr, Howard G, Mykkänen L, Tracy RP, Haffner SM: Chronic subclinical inflammation as part of the insulin resistance syndrome: the Insulin Resistance Atherosclerosis Study (IRAS). Circulation 2000, 102:42-47.

7. Pickup JC, Mattock MB, Chusney GD, Burt D: NIDDM as a disease of the innate immune system: association of acute-phase reactants and interleukin-6 with metabolic syndrome X. Diabetologia 1997 40:1286-1292.

8. Fernandez-Real JM, Vayreda M, Richart C, Gutierrez C, Broch M, Vendrell J, Ricart W: Circulating interleukin 6 levels, blood pressure, and insulin sensitivity in apparently healthy men and women. $J$ Clin Endocrinol Metab 2001, 86:1154-1159.

9. Arkan MC, Hevener AL, Greten FR, Maeda S, Li ZW, Long JM, Wynshaw-Boris A, Poli G, Olefsky J, Karin M: IKK- $\beta$ links inflammation to obesity-induced insulin resistance. Nat Med 2005, 11:191-198.

10. Romeo GR, Lee J, Shoelson SE: Metabolic syndrome, insulin resistance, and roles of inflammation-mechanisms and therapeutic targets. Arterioscler Thromb Vasc Biol 2012, 32:1771-1776.

11. Stagakis I, Bertsias G, Karvounaris S, Kavousanaki M, Virla D, Raptopoulou A, Kardassis D, Boumpas DT, Sidiropoulos Pl: Anti-tumor necrosis factor therapy improves insulin resistance, $\beta$ cell function and insulin signaling in active rheumatoid arthritis patients with high insulin resistance. Arthritis Res Ther 2012, 14:R141.

12. Feingold KR, Soued M, Staprans I, Gavin LA, Donahue ME, Huang BJ, Moser AH, Gulli R, Grunfeld C: Effect of tumor necrosis factor (TNF) on lipid metabolism in the diabetic rat: evidence that inhibition of adipose tissue lipoprotein lipase activity is not required for TNF-induced hyperlipidemia. J Clin Invest 1989, 83:1116-1121.

13. Hotamisligil GS, Shargill NS, Spiegelman BM: Adipose expression of tumor necrosis factor- $\alpha$ : direct role in obesity-linked insulin resistance. Science 1993, 259:87-91

14. Svenson KL, Lundqvist G, Wide L, Hällgren R: Impaired glucose handling in active rheumatoid arthritis: relationship to the secretion of insulin and counter-regulatory hormones. Metabolism 1987, 36:940-943.

15. Morohoshi M, Fujisawa K, Uchimura I, Numano F: Glucose-dependent interleukin 6 and tumor necrosis factor production by human peripheral blood monocytes in vitro. Diabetes 1996, 45:954-959.

16. Renier G, Clément I, Desfaits AC, Lambert A: Direct stimulatory effect of insulin-like growth factor-I on monocyte and macrophage tumor necrosis factor-a production. Endocrinology 1996, 137:4611-4618.

17. Soop M, Duxbury H, Agwunobi AO, Gibson JM, Hopkins SJ, Childs C, Cooper RG, Maycock P, Little RA, Carlson GL: Euglycemic hyperinsulinemia augments the cytokine and endocrine responses to endotoxin in humans. Am J Physiol Endocrinol Metab 2002, 282:E1276-E1285.

18. Kim SG, Ryu OH, Kim HY, Lee KW, Seo JA, Kim NH, Choi KM, Lee J, Baik SH, Choi DS: Effect of rosiglitazone on plasma adiponectin levels and arterial stiffness in subjects with prediabetes or non-diabetic metabolic syndrome. Eur J Endocrinol 2006, 154:433-440.

19. Aljada A, Garg R, Ghanim H, Mohanty P, Hamouda W, Assian E, Dandona P: Nuclear factor- $\mathrm{KB}$ suppressive and inhibitor- $\mathrm{KB}$ stimulatory effects of troglitazone in obese patients with type 2 diabetes: evidence of an antiinflammatory action? J Clin Endocrinol Metab 2001, 86:3250-3256.

20. Mohanty P, Aljada A, Ghanim H, Hofmeyer D, Tripathy D, Syed T, Al-Haddad W, Dhindsa S, Dandona P: Evidence for a potent antiinflammatory effect of rosiglitazone. J Clin Endocrinol Metab 2004, 89:2728-2735.

21. Ghanim H, Dhindsa S, Aljada A, Chaudhuri A, Viswanathan P, Dandona P: Low-dose rosiglitazone exerts an antiinflammatory effect with an increase in adiponectin independently of free fatty acid fall and insulin sensitization in obese type 2 diabetics. J Clin Endocrinol Metab 2006, 91:3553-3558.
22. Miyazaki Y, DeFronzo RA: Rosiglitazone and pioglitazone similarly improve insulin sensitivity and secretion, glucose tolerance and adipocytokines in type 2 diabetic patients. Diabetes Obes Metab 2008, 10:1204-1211.

23. Koufany M, Moulin D, Bianchi A, Muresan M, Sebillaud S, Netter P, Weryha $G$, Jouzeau JY: Anti-inflammatory effect of antidiabetic thiazolidinediones prevents bone resorption rather than cartilage changes in experimental polyarthritis. Arthritis Res Ther 2008, 10:R6.

24. Cuzzocrea S, Mazzon E, Dugo L, Patel NS, Serraino I, Di Paola R, Genovese T, Britti D, De Maio M, Caputi AP, Thiemermann C: Reduction in the evolution of murine type II collagen-induced arthritis by treatment with rosiglitazone, a ligand of the peroxisome proliferator-activated receptor $\gamma$. Arthritis Rheum 2003, 48:3544-3556.

25. Shahin D, Toraby EE, Abdel-Malek H, Boshra V, Elsamanoudy AZ, Shaheen D: Effect of peroxisome proliferator-activated receptor $\gamma$ agonist (pioglitazone) and methotrexate on disease activity in rheumatoid arthritis (experimental and clinical study). Clin Med Insights Arthritis Musculoskelet Disord 2011, 4:1-10.

26. Bongartz T, Coras B, Vogt T, Schölmerich J, Müller-Ladner U: Treatment of active psoriatic arthritis with the PPARy ligand pioglitazone: an openlabel pilot study. Rheumatology (Oxford) 2005, 44:126-129.

27. Juárez-Rojas JG, Medina-Urrutia AX, Jorge-Galarza E, Caracas-Portilla NA, Posadas-Sánchez R, Cardoso-Saldaña GC, Goycochea-Robles MV, Silveira LH, Lino-Pérez L, Mas-Oliva J, Pérez-Méndez O, Posadas-Romero C: Pioglitazone improves the cardiovascular profile in patients with uncomplicated systemic lupus erythematosus: a double-blind randomized clinical trial. Lupus 2012, 21:27-35.

28. McCoy RG, Irving BA, Soop M, Srinivasan M, Tatpati L, Chow L, Weymiller AJ, Carter RE, Nair KS: Effect of insulin sensitizer therapy on atherothrombotic and inflammatory profiles associated with insulin resistance. Mayo Clin Proc 2012, 87:561-570.

29. Arnett FC, Edworthy SM, Bloch DA, McShane DJ, Fries JF, Cooper NS, Healey LA, Kaplan SR, Liang MH, Luthra HS, Medsger TA Jr, Mitchell DM, Neustadt DH, Pinals RS, Jane G, Schaller JG, Sharp JT, Wilder RL, Hunder GG: The American Rheumatism Association 1987 revised criteria for the classification of rheumatoid arthritis. Arthritis Rheum 1988, 31:315-324.

30. European Medicines Agency: European Medicines Agency recommends new contra-indications and warnings for pioglitazone to reduce small increased risk of bladder cancer: benefit-risk balance remains positive in a limited population of type 2 diabetics. Press Release EMA/CHMP/568262/2011). London: Author; 21 July 2011. Available at http://www.ema.europa.eu/docs/en_GB/ document_library/Press_release/2011/07/WC500109176.pdf (accessed 13 September 2013).

31. US Food and Drug Administration (FDA): FDA Drug Safety Communication: Update to ongoing safety review of Actos (pioglitazone) and increased risk of bladder cancer. Safety Announcement). Silver Spring, MD: Author; 15 June 2011. Available at http://www.fda.gov/Drugs/DrugSafety/ucm259150.htm (accessed 13 September 2013.

32. Pincus T, Summey JA, Soraci SA Jr, Wallston KA, Hummon NP: Assessment of patient satisfaction in activities of daily living using a modified Stanford Health Assessment Questionnaire. Arthritis Rheum 1983, 26:1346-1353.

33. Matthews DR, Hosker JP, Rudenski AS, Naylor BA, Treacher DF, Turner RC: Homeostasis model assessment: insulin resistance and $\beta$-cell function from fasting plasma glucose and insulin concentrations in man. Diabetologia 1985, 28:412-419.

34. McCarey DW, Mclnnes IB, Madhok R, Hampson R, Scherbakov O, Ford Capell HA, Sattar N: Trial of Atorvastatin in Rheumatoid Arthritis (TARA): double-blind, randomised placebo-controlled trial. Lancet 2004 363:2015-2021.

35. Maini RN, Taylor PC, Szechinski J, Pavelka K, Bröll J, Balint G, Emery P, Raemen F, Petersen J, Smolen J, Thomson D, Kishimoto T, CHARISMA Study Group: Double-blind randomized controlled clinical trial of the interleukin-6 receptor antagonist, tocilizumab, in European patients with rheumatoid arthritis who had an incomplete response to methotrexate. Arthritis Rheum 2006, 54:2817-2829.

36. Baron RM, Kenny DA: The moderator-mediator variable distinction in social psychological research: conceptual, strategic, and statistical considerations. J Pers Soc Psychol 1986, 51:1173-1182.

37. Palma A, Sainaghi PP, Amoruso A, Fresu LG, Avanzi G, Pirisi M, Brunelleschi $S$ : Peroxisome proliferator-activated receptor- $\gamma$ expression in monocytes/ macrophages from rheumatoid arthritis patients: relation to disease 
activity and therapy efficacy--a pilot study. Rheumatology (Oxford) 2012, 51:1942-1952

38. Pepys MB, Hirschfield GM: C-reactive protein: a critical update. J Clin Invest 2003, 111:1805-1812.

39. Shedlovsky T, Scudder J: A comparison of erythrocyte sedimentation rates and electrophoretic patterns of normal and pathological human blood. J Exp Med 1942, 75:119-126.

40. Reinhart WH, Singh A, Straub PW: Red blood cell aggregation and sedimentation: the role of the cell shape. Br J Haematol 1989, 73:551-556.

41. Staniloae C, Mandadi V, Kurian D, Coppola J, Bernaski E, El-Khally Z, Morlote $M$, Pinassi E, Ambrose J: Pioglitazone improves endothelial function in non-diabetic patients with coronary artery disease. Cardiology 2007, 108:164-169.

42. Sarafidis PA, Lasaridis AN, Nilsson PM, Mouslech TF, Hitoglou-Makedou AD, Stafylas PC, Kazakos KA, Yovos JG, Tourkantonis AA: The effect of rosiglitazone on novel atherosclerotic risk factors in patients with type 2 diabetes mellitus and hypertension: an open-label observational study. Metabolism 2005, 54:1236-1242.

43. Schöndorf T, Lübben $G$, Hoopmann M, Borchert M, Forst T, Hohberg C, Löbig M, Armbruster FP, Roth W, Grabellus M, Pfützner A: Relaxin expression correlates significantly with serum fibrinogen variation in response to antidiabetic treatment in women with type 2 diabetes mellitus. Gynecol Endocrinol 2007, 23:356-360.

44. Aubert RE, Herrera V, Chen W, Haffner SM, Pendergrass M: Rosiglitazone and pioglitazone increase fracture risk in women and men with type 2 diabetes. Diabetes Obes Metab 2010, 12:716-721.

45. Azoulay L, Yin H, Filion KB, Assayaq J, Majdan A, Pollak MN, Suissa S: The use of pioglitazone and the risk of bladder cancer in people with type 2 diabetes: nested case-control study. BMJ 2012, 344:e3645.

46. Lincoff AM, Wolski K, Nicholls SJ, Nissen SE: Pioglitazone and risk of cardiovascular events in patients with type 2 diabetes mellitus: a metaanalysis of randomized trials. JAMA 2007, 298:1180-1188.

47. Hetzel J, Balletshofer B, Rittig K, Walcher D, Kratzer W, Hombach V, Häring HU, Koenig W, Marx N: Rapid effects of rosiglitazone treatment on endothelial function and inflammatory biomarkers. Arterioscler Thromb Vasc Biol 2005, 25:1804-1809.

48. Yki-Järvinen H: Thiazolidinediones. N Engl J Med 2004, 351:1106-1118

49. Guri AJ, Mohapatra SK, Horne WT 2nd, Hontecillas R, Bassaganya-Riera J: The role of T cell PPAR $y$ in mice with experimental inflammatory bowel disease. BMC Gastroenterol 2010, 10:60.

50. Jiang C, Ting AT, Seed B: PPAR- $\gamma$ agonists inhibit production of monocyte inflammatory cytokines. Nature 1998, 391:82-86.

51. Bright JJ, Kanakasabai S, Chearwae W, Chakraborty S: PPAR regulation of inflammatory signaling in CNS diseases. PPAR Res 2008, 2008:658520.

\section{doi:10.1186/ar4290}

Cite this article as: Ormseth et al: Peroxisome proliferator-activated receptor $\gamma$ agonist effect on rheumatoid arthritis: a randomized controlled trial. Arthritis Research \& Therapy 2013 15:R110.

\section{Submit your next manuscript to BioMed Central and take full advantage of:}

- Convenient online submission

- Thorough peer review

- No space constraints or color figure charges

- Immediate publication on acceptance

- Inclusion in PubMed, CAS, Scopus and Google Scholar

- Research which is freely available for redistribution

Submit your manuscript at www.biomedcentral.com/submit 